\title{
Partial cystectomy for urothelial carcinoma of the bladder: Practice patterns and outcomes in the general population
}

\author{
Michael J. Leveridge, MD ${ }^{1,2}$; D. Robert Siemens, MD ${ }^{1,2}$; Jason Izard, MD, MPH ${ }^{1,2}$; \\ Xuejiao Wei, $\mathrm{MSc}^{4}$; Christopher M. Booth, $\mathrm{MD}^{2,3,4}$ \\ ${ }^{1}$ Departments of Urology; ${ }^{2}$ Oncology; ${ }^{3}$ Public Health Sciences; ${ }^{4}$ Division of Cancer Care and \\ Epidemiology, Queen’s University Cancer Research Institute; Queen’s University, Kingston, ON, Canada
}

Acknowledgements: Parts of this material are based on data and information provided by Cancer Care Ontario; however, the analysis, conclusions, opinions and statements expressed herein are those of the authors and not necessarily those of Cancer Care Ontario.

This study was supported by the Institute for Clinical Evaluative Sciences (ICES), which is funded by an annual grant from the Ontario Ministry of Health and Long-Term Care (MOHLTC). The opinions, results and conclusions reported in this paper are those of the authors and are independent from the funding sources. No endorsement by ICES or the Ontario MOHLTC is intended or should be inferred.

Dr. Booth had full access to all the data in the study and takes responsibility for the integrity of the data and the accuracy of the data analysis.

Funding: Dr. Booth is supported as a Canada Research Chair in Population Cancer Care. This work was also supported by the Canada Foundation for Innovation and Cancer Care Ontario.

Cite as: Can Urol Assoc J 2017; Epub ahead of print.

http://dx.doi.org/10.5489/cuaj.4403

Published online November 1, 2017

$* * *$

\section{Abstract}

Introduction: Partial cystectomy (PC) for urothelial carcinoma (UC) in selected patients may avoid the morbidity of radical cystectomy (RC). We describe utilization and outcomes of PC for UC in routine clinical practice.

Methods: All patients with urothelial carcinoma of the bladder (UCB) undergoing PC or RC in Ontario from 1994-2008 were identified using the Ontario Cancer Registry and linked electronic records. Pathology reports were reviewed. Variables associated with PC use were identified using logistic regression. Cox proportional hazards model identified factors affecting cancer-specific (CSS) and overall survival (OS).

Results: A total of 3320 patients underwent PC $(n=181 ; 5 \%)$ or RC ( $n=3139 ; 95 \%)$ from 1994-2008. PC patients were older (36\% 80+ years vs. $19 \%$; $\mathrm{p}<0.001$ ) and more likely to have organ-confined (<pT3) disease (54\% vs. 36\% RC; $<<0.001)$. Two-thirds $(67 \%)$ of 
PC patients did not undergo lymph node dissection ( $24 \%$ for $\mathrm{RC} ; \mathrm{p}<0.001)$. Factors associated with having PC included older age (odds ratio [OR] 1.55; 95\% confidence interval [CI] 0.96-2.51 for 70+ years), moderate comorbidity (OR 1.95; 95\% CI 1.133.37), and surgery outside of a comprehensive cancer centre (OR 1.44; 95\% CI 1.032.01). Unadjusted five-year OS for PC and RC cases was $34 \%$ and $33 \%$, respectively ( $\mathrm{p}=0.455)$; CSS at five years was $43 \%$ and $37 \%(\mathrm{p}=0.045)$. On adjusted analysis, PC was associated with comparable CSS (hazard ratio [HR] 0.87, 95\% CI 0.70-1.09) and OS (HR 0.95, 95\% CI 0.79-1.14) as RC.

Conclusions: In routine clinical practice, PC is not common. A substantial proportion of patients treated with PC achieve long-term survival. PC remains a treatment option in selected patients with UCB.

\section{Introduction}

Bladder cancer is a disease of the elderly who often have concomitant frailty and comorbidity[1]. Radical cystectomy (RC), long the standard management of muscleinvasive bladder cancer (MIBC), is a morbid procedure with a major complication rate even in high-volume centers of over 30\%[2]. We have previously reported that among patients older than 80 years, 90-day postoperative mortality following radical cystectomy is $15 \%$. Moreover, 5-year overall survival and cancer-specific survival are lower than in younger patients[3]. The morbidity of major exenterative and reconstructive surgery has led to the use of bladder-sparing techniques and protocols for some patients with MIBC, including maximal transurethral resection, radiation and chemotherapy[4,5]. However, many patients are not candidates for chemotherapy and therefore ineligible for optimal bladder sparing approaches[6]. Partial cystectomy (PC), ideally in conjunction with pelvic lymph node dissection (PLND), remains an option for highly selected patients with MIBC.

A handful of published reports have suggested that PC has comparable oncologic and survival outcomes to RC, however these reports predominately come from singlecenter series[7-9]. Partial cystectomy data often includes non-urothelial cancers and are therefore difficult to compare with radical cystectomy. Only a handful of studies have described utilization and outcome of PC in routine clinical practice[7,10,11]. We undertook a population-based retrospective cohort study to describe the use and outcomes of PC in the Canadian province of Ontario. 


\section{Methods}

\section{Study design and population}

This is a secondary report of a population-based, retrospective cohort study of all patients with bladder cancer treated with cystectomy in the Canadian province of Ontario.

Detailed methodology and primary results have been reported elsewhere[12]. Ontario has a single-payer universal health insurance program that covers a population of approximately 13.5 million. All incident cases of urothelial bladder cancer in Ontario who underwent cystectomy in 1994-2008 were identified using the Ontario Cancer Registry and linked treatment records. Surgical pathology reports were obtained for all cystectomy cases.

The primary objectives of this study were to describe the utilization and outcomes of PC in the surgical management of UCB at the population level, and compare these to patients undergoing RC. Ethics approval was obtained from the Research Ethics Board of Queen’s University.

\section{Data sources}

The Ontario Cancer Registry (OCR) is a passive registry that captures diagnostic and demographic information on at least $98 \%$ of all incident cases of cancer diagnosed in the province of Ontario[13]. The following data was obtained via the OCR: International Classification of Disease, version 9 (ICD-9) code; the ICD-O histology code; date of diagnosis; date of birth; place of residence at diagnosis; vital status; date of death; and cause of death. Vital status was available up to December 31, 2010 and cause of death was available up to December 31, 2008. Socioeconomic status (SES) data of the community in which patients resided at time of diagnosis were linked to the OCR as described previously, divided into quintiles (Q) of median household income as compared to the entire province[14]. Q1 represents the communities where the poorest $20 \%$ of the Ontario population resided. Hospitalization records obtained from the Canadian Institute for Health Information (CIHI) included surgical interventions, length of stay and repeat admission to hospital. Hospital participation in collection of records is consistent and complete throughout Ontario[15]. Chemotherapy use was identified through provincial physician billing records and treatment records from Ontario's regional cancer centers. Surgical pathology reports were obtained from the Ontario Cancer Registry (OCR). An electronic database was created by a team of trained data abstractors who reviewed and abstracted data from the pathology reports. In this study we use pathologic (not clinical) stage.

Comorbidity was classified by the Charlson Comorbidity Index, based on noncancer diagnoses recorded within 5 years of surgery[16]. Neoadjuvant chemotherapy (NACT) was defined as occurring $\leq 16$ weeks before surgery, and adjuvant chemotherapy (ACT) within 16 weeks following surgery. Pre-operative radiotherapy (RT) was defined 
as RT giving within 16 weeks before cystectomy; post-operative RT was given within 16 weeks after cystectomy. RT beyond 16 weeks from surgery was considered to be salvage RT.

\section{Statistical analysis}

Overall (OS) and cancer-specific (CSS) survival were determined from date of surgery using the Kaplan-Meier technique and comparisons between groups were made using the log-rank test. Factors associated with PC were evaluated with logistic regression analyses. The association between patient-, disease-, and treatment-related factors with overall/cancer-specific survival was evaluated using the Cox proportional hazards regression model. The survival analyses were restricted to patients that did not receive NACT or pre-operative radiotherapy, because information about pathologic stage for these patients would be less reliable. As per institutional privacy policy, patient subgroups with $<6$ cases are suppressed to preserve patient confidentiality. Results were considered statistically significant at $\mathrm{p}$-value $<0.05$. As per institutional privacy policy we do not report any data with cell sizes $<6$. All analyses were performed using SAS version 9.3 (SAS Institute, Cary, NC).

\section{Results}

\section{Study population}

We identified 3320 patients who underwent PC or RC for urothelial cancer of the bladder in Ontario between 1994 and 2008. Five percent (181/3320) of cases underwent PC. Characteristics of the study population are shown in Table 1. A higher proportion of PC vs RC patients were octogenarians (36\% vs $19 \%, \mathrm{p}<0.001)$. PC patients were more likely to have organ-confined ( $<\mathrm{pT} 3$ ) disease ( $54 \%$ vs $36 \%, \mathrm{p}<0.001)$ than patients with RC. Two thirds of patients with PC (121/181, 67\%) did not have lymph nodes resected at the time of surgery; conversely, 24\% (741/3139, $\mathrm{p}<0.001)$ of patients with RC were NX. Surgical margins were positive in a higher proportion of PC patients compared to patients with total cystectomy ( $18 \%$ vs $12 \%$, $\mathrm{p}=0.013)$.

\section{Practice patterns}

Use of peri-operative chemotherapy was less common among patients treated with PC. Eighteen percent (553/3139) of patients with total cystectomy had adjuvant chemotherapy compared to $12 \%(22 / 181)$ of PC patients ( $\mathrm{p}=0.059)$. Neoadjuvant chemotherapy was delivered to $4 \%$ of cases with total cystectomy (128/3139); $1 \%$ $(<6 / 181)$ patients with PC received neoadjuvant chemotherapy $(\mathrm{p}=0.009)$. Post-operative RT [3\% (6/181) vs $1 \%$ (23/3319), p=0.004] and salvage RT [4\% (7/181) vs $2 \%$ (61/3319), p=0.096] was more common among patients with PC compared to RC.

Variables associated with the use of partial cystectomy are shown in Table 2. Use of PC has decreased over time $(\mathrm{p}<0.001)$. On adjusted analyses, patients $70+$ years of age 
(OR 1.55, 95\%CI 0.96-2.51) and those with greater co-morbidity (OR 1.95, 95\%CI 1.133.37) were more likely to have PC. Patients who underwent surgery at a comprehensive cancer center were less likely to have PC (OR 0.70, 95\%CI 0.50-0.97). More advanced tumours (T3-4) were less likely to be managed with PC (OR 0.49, 95\%CI 0.36-0.67). There was no significant regional variation in use of PC. Gender was not associated with use of PC. No patients with PC subsequently underwent salvage cystectomy in our data. Fewer than 5\%of patients underwent salvage RC after PC in this cohort.

\section{Outcomes}

Outcomes of patients treated with PC and RC are shown in Table 3. Median length of stay was shorter for PC patients (8 days vs 11 days; $\mathrm{p}<0.001$ ). Readmission rate and early postoperative mortality were similar between groups. Unadjusted five-year OS for PC and RC cases was 34\% and 33\% respectively ( $\mathrm{p}=0.455)$; CSS at 5 years was $43 \%$ and $37 \%(\mathrm{p}=0.045)$.

Adjusted overall and cancer-specific survival analyses for patients treated with PC are shown in Table 4. Among patients treated with PC, more advanced disease (T stage and N stage) and LVI were associated with inferior survival; age, co-morbidity, and margin status were not associated with outcome.

Adjusted survival analyses for all patients having cystectomy (partial and radical) are shown in Table 5. Among PC and RC patients greater age and co-morbidity are associated with inferior survival; $\mathrm{T}$ stage, $\mathrm{N}$ stage, and lymphovascular invasion are also associated with inferior outcomes. Adjusted analyses do not suggest any significant difference in CSS (HR 0.87, 95\%CI 0.70-1.09) or OS (HR 0.95, 95\%CI 0.79-1.14) among patients treated with partial cystectomy versus radical cystectomy.

\section{Discussion}

In this study we describe utilization of partial cystectomy for urothelial cancer of the bladder and outcomes achieved in routine clinical practice. Several important findings have emerged. First, PC is not common in the general population and utilization has decreased over time. In the most recent study (2004-2008) era only 4\% of patients treated with cystectomy had PC. Second, factors associated with having PC include older age, greater co-morbidity, less extensive disease, and treatment outside a comprehensive cancer center. Third, $67 \%$ of patients with PC did not have lymph nodes resected at the time of surgery. Fourth, among patients treated with PC, T stage, N stage and LVI are associated with survival. Finally, in adjusted analyses, the long-term survival of patients treated with PC is comparable to those treated with RC.

Partial cystectomy is an understandably attractive option for patients with MIBC as compared to a radical cystectomy given the less disruptive surgical procedure and the functional bladder left in situ. PC is associated with earlier post-surgical recovery, avoidance of stigma and management issues that may accompany urinary diversion and 
the possible retention of sexual function[11,17]. PC is likely only feasible in a small proportion of MIBC patients (less than 10\%), as indications are limited to those with a small solitary mass favorably located to allow adequate post-operative capacity, away from the trigone and in the absence of associated carcinoma in situ[8,11].

Partial cystectomy for UCB is relatively uncommon in the province of Ontario, and its use appears to be decreasing in more recent years. Several other series have observed similar rates of PC. Fedeli et al noted a decrease in PC as a proportion of all cystectomies in Italian centers, from 5.5\% during 2000-2002 to 3.3\% during 20062008[18]. Two population-level studies from the United States and from the Canadian province of Quebec found 18-30\% of patients underwent PC, however these studies may have included non-urothelial histologies.[10,11]. Moreover, because these studies classified cystectomy based on administrative data sources, they may over-estimate the use of PC due to misclassification bias. Extent of cystectomy in the present study was established based on review of the surgical pathology report. Moreover, our study assessed only urothelial cancer. Removing other less aggressive or benign histology provides more clarity into the outcomes of PC in this context.

It is perhaps unsurprising that patients chosen for partial cystectomy are older and greater comorbidity than RC patients. However, previous population level data using the Surveillance Epidemiology and End Results (SEER) database have shown inconsistent effects of age, gender and comorbidity on the choice to use PC[7,11]. Our results also suggest that PC is more often used in patients with less extensive disease. This, as well as the generally more elderly and unwell patients, may explain the decreased use of adjuvant chemotherapy in PC patients. These population data show a slightly higher local stage at PC than prior published population and single-center series, likely reflecting the analysis of urothelial cancer in isolation, while suggesting differences in patient selection or time to surgery in the population at large[8,9,19-21]. It is notable that no lymph node pathologic data was available for $67 \%$ of PC cases in our series (and $24 \%$ of RC), suggesting that PLND was not considered part of the surgical procedure, possibly to decrease operative time and/or morbidity. Prior studies have shown highly variable use of PLND in other settings[8,10,20]. It is clear that PLND is a critical component of definitive surgical management of MIBC from a risk stratification and prognostic perspective, and these data point to a deficit in current practice[3,22,23]. The decision to forego PLND at partial cystectomy, given its relatively benign nature, may compromise long-term outcome in these patients.

Adjusted analyses in our study suggest that patients who have PC have comparable survival to RC. These data however must be interpreted in light of the fact that age, comorbidity, and extent of disease were very different between the 2 groups and the analyses are therefore prone to residual confounding. Patients with PC were older than those with RC; this might explain the fact that PC was not associated with any 
substantial reduction in post-operative re-admission rates or mortality. The low rate of lymph node dissection is also notable and suggests the need for future quality improvement initiatives. Outcomes observed in our cohort of PC patients are inferior to single-center series which have reported $58-70 \%$ OS at 5 years, and $65-87 \%$ DSS, as compared to $34 \%$ and $43 \%$, respectively in our study. This likely reflects selection bias and the different case mix of patients treated in single centers compared to care in the general population.[8,9,19,20].

Our results need to be considered in the context of study limitations. As with all retrospective cohort studies, our results are prone to bias by confounding. Although the pathology reports were individually reviewed by a trained team of abstractors, surgical specimens did not undergo central pathology review. While our existing data-sets allow us to describe practice and outcomes for all patients treated with cystectomy in Ontario, we do not have granular details regarding patient performance status, previous transurethral resection history, location of disease, tumour size, carcinoma-in-situ or bladder/renal function. This limits our ability to evaluate appropriateness of case selection for PC. We also do not have information on date of disease recurrence so cannot describe relapse-free survival.

This database has significant strengths as compared to other population data sets. Detailed pathologic information from abstracted reports and complete chemotherapy data are distinguishing characteristics that provide a uniquely comprehensive window into routine urologic practice in the general population.

\section{Conclusion}

The decision to choose PC over RC in patients with UCB is complex and relates to disease characteristics and patient preference. While partial cystectomy is not used commonly in the general population, adjusted outcomes suggest that survival is comparable to RC. Our data suggest that lymph node dissection may be underutilized in PC. 


\section{References}

1. Shariat SF, Milowsky M, Droller MJ. Bladder cancer in the elderly. Urol Oncol. 2009;27(6):653-667

2. Stein JP, Lieskovsky G, Cote R, et al. Radical cystectomy in the treatment of invasive bladder cancer: long-term results in 1,054 patients. J Clin Oncol. 2001;19(3):666-675

3. Leveridge MJ, Siemens DR, Mackillop WJ, et al. Radical cystectomy and adjuvant chemotherapy for bladder cancer in the elderly: a population-based study. Urology. 2015;85(4):791-798

4. Ploussard G, Daneshmand S, Efstathiou JA, et al. Critical analysis of bladder sparing with trimodal therapy in muscle-invasive bladder cancer: a systematic review. Eur Urol. 2014;66(1):120-137

5. James ND, Hussain SA, Hall E, et al. Radiotherapy with or without chemotherapy in muscle-invasive bladder cancer. N Engl J Med. 2012;366(16):1477-1488

6. Dash A, Galsky MD, Vickers AJ, et al. Impact of renal impairment on eligibility for adjuvant cisplatin-based chemotherapy in patients with urothelial carcinoma of the bladder. Cancer. 2006;107(3):506-513

7. Capitanio U, Isbarn H, Shariat SF, et al. Partial cystectomy does not undermine cancer control in appropriately selected patients with urothelial carcinoma of the bladder: a population-based matched analysist. Urology. 2009;74(4):858-864

8. Holzbeierlein JM, Lopez-Corona E, Bochner BH, et al. Partial cystectomy: a contemporary review of the memorial sloan-kettering cancer center experience and recommendations for patient selection. The Journal of Urology. 2004;172(3):878881

9. Ma B, Li H, Zhang C, et al. Lymphovascular invasion, ureteral reimplantation and prior history of urothelial carcinoma are associated with poor prognosis after partial cystectomy for muscle-invasive bladder cancer with negative pelvic lymph nodes. Eur J Surg Oncol. 2013;39(10):1150-1156

10. Fahmy N, Aprikian A, Tanguay S, et al. Practice patterns and recurrence after partial cystectomy for bladder cancer. World J Urol. 2010;28(4):419-423

11. Hollenbeck BK, Taub DA, Dunn RL et al. Quality of care: partial cystectomy for bladder cancer--a case of inappropriate use? J Urol. 2005;174(3):1050-4

12. Booth CM, Siemens DR, Li G, et al. Perioperative chemotherapy for muscleinvasive bladder cancer: A population-based outcomes study. Cancer. 2014;120(11):1630-1638

13. Clarke EA, Marrett LD, Kreiger N. Appendix 3 (c) Cancer registration in Ontario: a computer approach. Cancer Registration: Principles and Methods. Jensen OM, Parkin R, MacLennan R, Muir CS, Skeet RG Eds. International Agency for Research on Cancer, Lyon, France 1991 
14. Booth CM, Li G, Zhang-Salomons J et al. The impact of socioeconomic status on stage of cancer at diagnosis and survival. Cancer 2010;116:4160-7

15. Goel V, Williams JI, Anderson GM et al, Eds. ICES Practice Atlas 1996. Canadian Medical Association, Ottawa, Canada 1996

16. Charlson ME, Pompei P, Ales KL et al. A new method of classifying prognostic comorbidity in longitudinal studies: development and validation. J Chronic Dis. 1987;40(5):373-383

17. Knoedler J, Frank I. Organ-sparing surgery in urology: partial cystectomy. Curr Opin Urol. 2015;25(2):111-115

18. Fedeli U, Novara G, Galassi C, et al. Population-based analyses of radical cystectomy and urinary diversion for bladder cancer in northern Italy. BJU Int. 2011;108(8 Pt 2):E266-E271

19. Kassouf W, Swanson D, Kamat AM, et al. Partial Cystectomy for Muscle Invasive Urothelial Carcinoma of the Bladder: A Contemporary Review of the M. D. Anderson Cancer Center Experience. The Journal of Urology. 2006;175(6):20582062

20. Smaldone MC, Jacobs BL, Smaldone AM et al. Long-term results of selective partial cystectomy for invasive urothelial bladder carcinoma. Urology. 2008;72(3):613-616

21. Knoedler JJ, Boorjian SA, Kim SP, et al. Does partial cystectomy compromise oncologic outcomes for patients with bladder cancer compared to radical cystectomy? A matched case-control analysis. J Urol. 2012;188(4):1115-1119

22. Leissner J, Ghoneim MA, Abol-Enein $\mathrm{H}$, et al. Extended radical lymphadenectomy in patients with urothelial bladder cancer: results of a prospective multicenter study. J Urol. 2004;171(1):139-144

23. Tilki D, Brausi M, Colombo R, et al. Lymphadenectomy for bladder cancer at the time of radical cystectomy. Eur Urol. 2013;64(2):266-276 
Figures and Tables

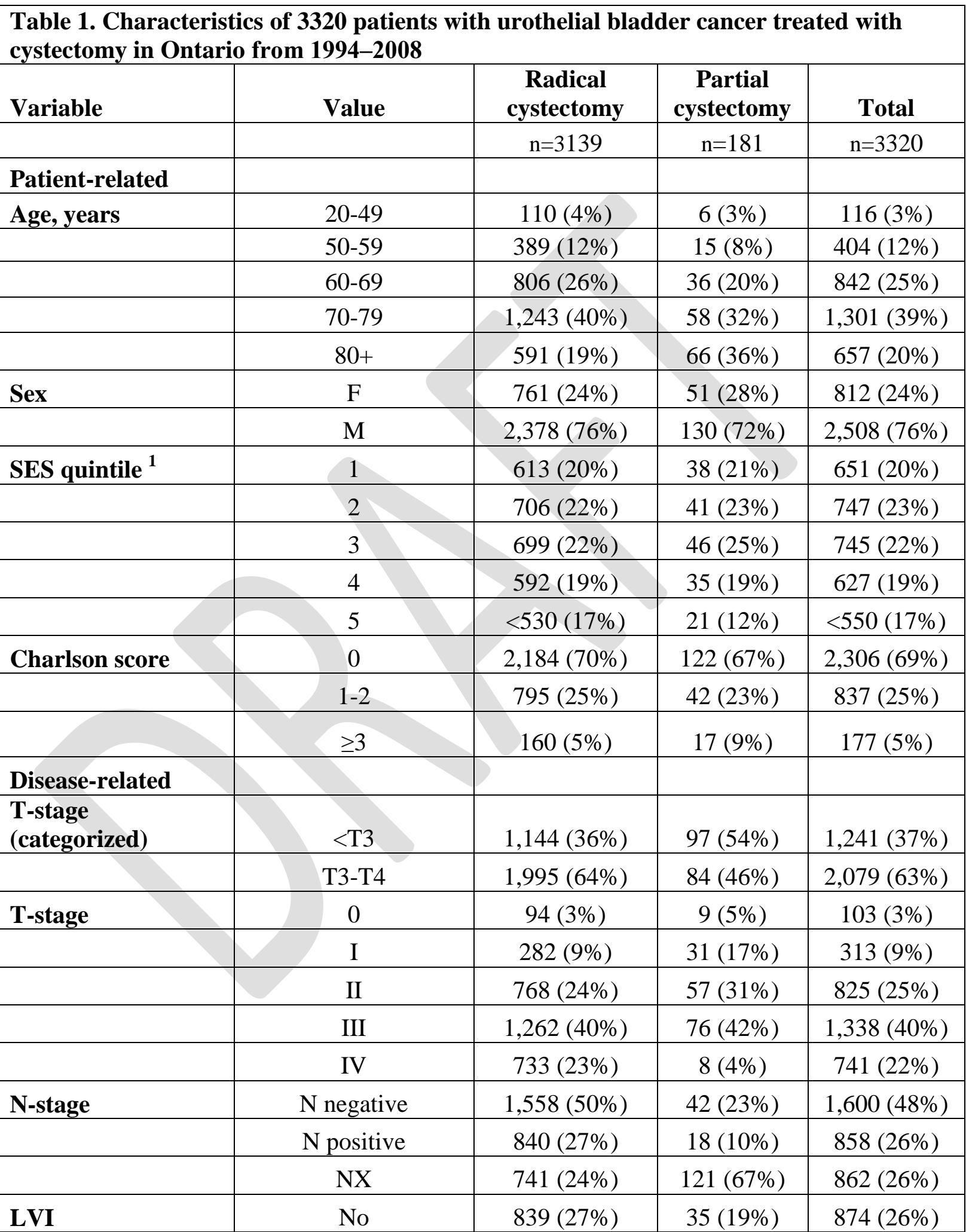




\begin{tabular}{|l|c|c|c|c|}
\hline & Yes & $1,485(47 \%)$ & $68(38 \%)$ & $1,553(47 \%)$ \\
\hline & Unstated & $815(26 \%)$ & $78(43 \%)$ & $893(27 \%)$ \\
\hline Margin status & Negative/unstated & $2,762(88 \%)$ & $148(82 \%)$ & $2,910(88 \%)$ \\
\hline & Positive & $377(12 \%)$ & $33(18 \%)$ & $410(12 \%)$ \\
\hline
\end{tabular}

${ }^{1}$ SES quintile 1 represents patients from the poorest communities in Ontario. SES data were not available for $<6$ patients.

Table 2. Variables associated with use of partial cystectomy (PC) among patients with urothelial bladder cancer treated with cystectomy in Ontario, 1994-2008 $\left(\mathrm{n}=3185^{*}\right)$

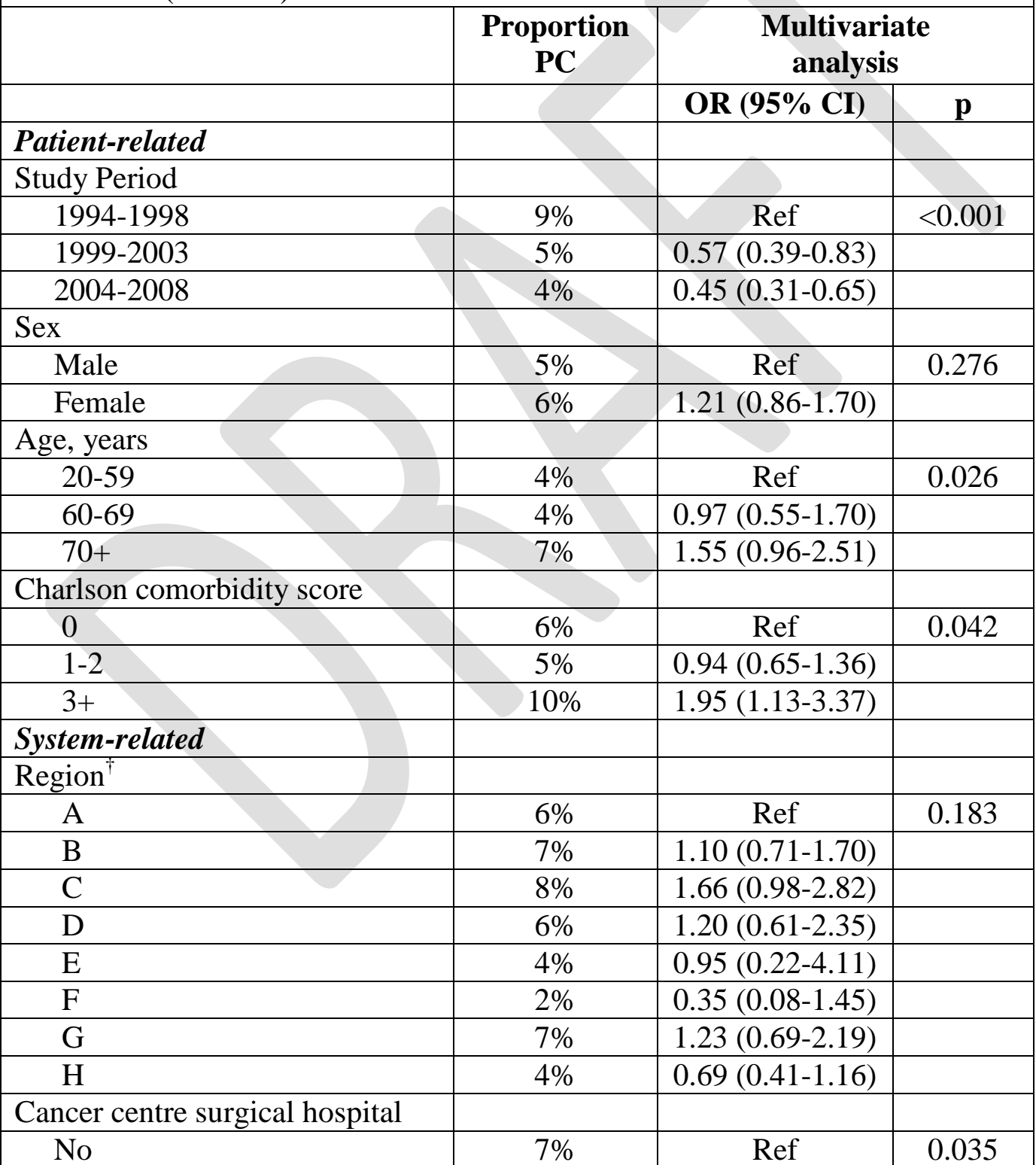




\begin{tabular}{|l|c|c|c|}
\hline Yes & $4 \%$ & $0.70(0.50-0.97)$ & \\
\hline Disease-related & & & \\
\hline Pathologic T stage & & & \\
\hline$<$ T3 & $8 \%$ & Ref & $<0.001$ \\
\hline T3-4 & $4 \%$ & $0.49(0.36-0.67)$ & \\
\hline
\end{tabular}

135 patients with NACT and/or pre-operative RT are removed from the analysis since T stage does not reflect actual surgical pathologic stage. ${ }^{\dagger}$ Region data were not available for $<6$ patients.

\begin{tabular}{|l|c|c|c|}
\hline \multicolumn{4}{|l|}{$\begin{array}{l}\text { Table 3. Unadjusted outcomes of patients with urothelial bladder cancer } \\
\text { treated with partial or radical cystectomy in Ontario, 1994-2008 (n=3320) }\end{array}$} \\
\hline & $\begin{array}{c}\text { Partial } \\
\text { n=181 }\end{array}$ & $\begin{array}{c}\text { Radical } \\
\text { n=3139 }\end{array}$ & p \\
\hline Outcome & \multicolumn{3}{|c|}{} \\
\hline Mean/median LOS (days) & $11 / 8$ & $15 / 11$ & $<0.001$ \\
\hline 30 day re-admission N (\%) & $21(12 \%)$ & $494(16 \%)$ & 0.135 \\
\hline 90 day re-admission N \%) & $43(24 \%)$ & $1017(32 \%)$ & 0.015 \\
\hline 30 day mortality N $(\%)$ & $<=5(<3 \%)$ & $79(3 \%)$ & 0.196 \\
\hline 90 day mortality N (\%) & $8(4 \%)$ & $263(8 \%)$ & 0.059 \\
\hline 5 year OS (95\%CI)* & $34 \%(27-41 \%)$ & $33 \%(31-35 \%)$ & 0.455 \\
\hline 5 year CSS (95\%CI) & $43 \%(34-51 \%)$ & $37 \%(35-39 \%)$ & 0.045 \\
\hline
\end{tabular}

${ }^{\dagger} \mathrm{p}$ values for 5 year OS and CSS are based on log-rank test. ${ }^{*}$ p value for mean LOS is Kruskal Wallis test. Others are Chi-square test. Fisher's exact test was used for 30-day mortality because expected cell size was $<5$. 


\begin{tabular}{|c|c|c|c|c|}
\hline \multirow[t]{3}{*}{ Characteristic } & \multicolumn{2}{|c|}{ CSS } & \multicolumn{2}{|l|}{ OS } \\
\hline & \multicolumn{2}{|c|}{ Multivariate analysis } & \multicolumn{2}{|c|}{ Multivariate analysis } \\
\hline & HR (95\% CI) & p trend & HR (95\% CI) & p trend \\
\hline \multicolumn{5}{|l|}{ Patient-related } \\
\hline Age, years & & 0.414 & & 0.112 \\
\hline $20-59(n=21)$ & Ref & & Ref & \\
\hline $60-69(n=35)$ & $1.63(0.72-3.70)$ & 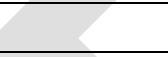 & $1.21(0.61-2.43)$ & \\
\hline $70+(n=124)$ & $1.62(0.78-3.37)$ & 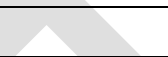 & $1.74(0.94-3.22)$ & \\
\hline Co-morbidity score & & 0.462 & & 0.252 \\
\hline $0(\mathrm{n}=57)$ & Ref & & Ref & \\
\hline$>0(n=15)$ & $1.18(0.76-1.85)$ & & $1.24(0.86-1.78)$ & \\
\hline \multicolumn{5}{|l|}{ Disease-related } \\
\hline T stage & 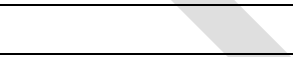 & 0.002 & & 0.040 \\
\hline$<\mathrm{T} 3(\mathrm{n}=96)$ & Ref & 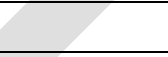 & Ref & \\
\hline T3-T4 (n=84) & $2.10(1.32-3.34)$ & 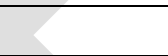 & $1.49(1.02-2.19)$ & \\
\hline N stage & & 0.077 & & 0.001 \\
\hline $\mathrm{N}$ negative $(\mathrm{n}=42)$ & Ref & 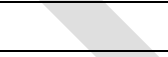 & Ref & \\
\hline $\mathrm{N}$ positive $(\mathrm{n}=17)$ & $2.43(1.11-5.31)$ & & $3.45(1.80-6.63)$ & \\
\hline NX $(n=121)$ & $1.56(0.86-2.83)$ & + & $1.49(0.92-2.41)$ & \\
\hline LVI & 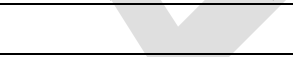 & 0.011 & & 0.024 \\
\hline No $(n=35)$ & Ref & & Ref & \\
\hline Yes $(n=68)$ & $2.00(1.03-3.87)$ & & $1.49(0.90-2.46)$ & \\
\hline Unstated $(\mathrm{n}=77)$ & $0.94(0.49-1.82)$ & & $0.83(0.50-1.36)$ & \\
\hline Margin status & $+2+y$ & 0.548 & & 0.380 \\
\hline Negative/unstated $(\mathrm{n}=147)$ & Ref & & Ref & \\
\hline Positive $(n=33)$ & $0.84(0.47-1.48)$ & & $1.22(0.78-1.90)$ & \\
\hline
\end{tabular}

${ }^{*} \leq 5$ cases with NACT and/or pre-op RT were excluded from this analysis as T and $\mathrm{N}$ stage at time of surgery would not accurately reflect extent of disease. 


\begin{tabular}{|c|c|c|c|c|}
\hline \multirow[t]{3}{*}{ Characteristic } & \multirow{2}{*}{\multicolumn{2}{|c|}{$\begin{array}{c}\text { CSS } \\
\text { Multivariate analysis } \\
\end{array}$}} & \multicolumn{2}{|l|}{ OS } \\
\hline & & & Multivariate a & ysis \\
\hline & HR (95\% CI) & p trend & HR (95\% CI) & p trend \\
\hline Age, years & & $<0.001$ & & $<0.001$ \\
\hline $20-59(n=480)$ & Ref & 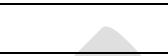 & Ref & \\
\hline $60-69(n=804)$ & $1.11(0.94-1.30)$ & 2 & $1.21(1.04-1.40)$ & \\
\hline $70+(n=1901)$ & $1.39(1.20-1.61)$ & 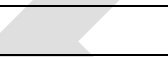 & 1.64 (1.44-1.87) & \\
\hline Comorbidity score & & 0.003 & & $<0.001$ \\
\hline $0(n=2198)$ & Ref & & Ref & \\
\hline$>0(n=987)$ & $1.17(1.05-1.29)$ & 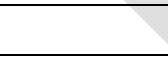 & $1.28(1.17-1.40)$ & \\
\hline T stage & & $<0.001$ & & $<0.001$ \\
\hline$<\mathrm{T} 3(\mathrm{n}=1185)$ & Ref & & Ref & \\
\hline T3-T4 $(n=2000)$ & $2.01(1.79-2.25)$ & & $1.83(1.66-2.01)$ & \\
\hline $\mathrm{N}$ stage & & $<0.001$ & +2 & $<0.001$ \\
\hline N negative $(n=1529)$ & Ref & & Ref & \\
\hline $\mathrm{N}$ positive $(\mathrm{n}=825)$ & $1.58(1.40-1.78)$ & & 1.52 (1.37-1.69) & \\
\hline NX $(n=831)$ & $1.41(1.26-1.59)$ & +2 & $1.42(1.28-1.58)$ & \\
\hline LVI & 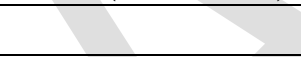 & $<0.001$ & & $<0.001$ \\
\hline No $(n=830)$ & Ref & & Ref & \\
\hline Yes $(n=1497)$ & $2.08(1.81-2.39)$ & 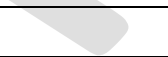 & $1.79(1.60-2.02)$ & \\
\hline Unstated $(n=858)$ & $1.31(1.13-1.52)$ & & $1.20(1.06-1.36)$ & \\
\hline Surgery & 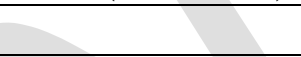 & 0.224 & & 0.568 \\
\hline Total cystectomy $(n=3005)$ & Ref & & Ref & \\
\hline Partial cystectomy $(n=180)$ & $0.87(0.70-1.09)$ & & $0.95(0.79-1.14)$ & \\
\hline
\end{tabular}

* 135 cases with NACT and/or pre-op RT were excluded from this analysis as T and N stage at time of surgery would not accurately reflect extent of disease. 